\title{
Symbolic dynamics for a Sierpinski curve Julia set
}

\author{
ROBERT L. DEVANEY* and DANIEL M. LOOK \\ Department of Mathematics, Boston University, 111 Cummington Street, Boston MA 02215, USA
}

(Received 22 February 2004; in final form 24 September 2004)

\begin{abstract}
In this paper we investigate the dynamics of certain rational functions on their Julia sets. We pay particular attention to the case where the Julia set is a Sierpinski curve. In this case, any two such Julia sets are known to be homeomorphic. However, the dynamics on these sets are often quite different. In this paper, we use symbolic dynamics to show how these different dynamical behaviors may be analyzed.

Keywords: Julia sets; Rational maps; Sierpinski curves
\end{abstract}

\section{Introduction}

In this paper, we discuss the dynamics of certain members of the family of rational maps of the complex plane given by

$$
F_{\lambda}(z)=z^{2}+\frac{\lambda}{z^{2}}
$$

Of particular interest is the dynamical behavior of these maps on the Julia set of $F_{\lambda}$, which we denote by $J\left(F_{\lambda}\right)$. The following Theorem was proved in [4]:

THEOREM There are infinitely many open sets $\mathcal{O}_{j}$ in the $\lambda$-plane for this family having the following properties:

1. For each $\lambda \in \mathcal{O}_{j}$, the Julia set of $F_{\lambda}$ is a Sierpinski curve; as a consequence, any two of these Julia sets are homeomorphic.

2. However, if $\lambda \in \mathcal{O}_{j}$ and $\mu \in \mathcal{O}_{k}$ with $j \neq k$, then $F_{\lambda}$ is not topologically conjugate to $F_{\mu}$ on their respective Julia sets.

A Sierpinski curve is a planar set that is homeomorphic to the well known Sierpinski carpet fractal (see figure 2). More generally, it is known [9] that any planar set that is compact, connected, nowhere dense, locally connected, and has the property that any two complementary domains are bounded by mutually disjoint simple closed curves is a Sierpinski curve. See also [10,13].

*Corresponding author. Email: bob@bu.edu

Journal of Difference Equations and Applications

ISSN 1023-6198 print/ISSN 1563-5120 online (C) 2005 Taylor \& Francis Group Ltd

http://www.tandf.co.uk/journals

DOI: $10.1080 / 10236190412331334473$ 


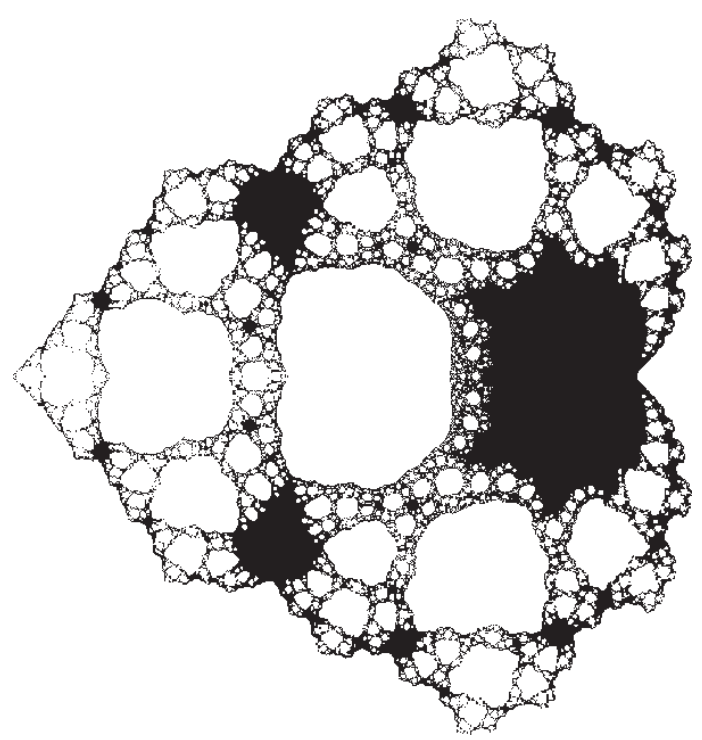

Figure 1. The parameter plane for the family $F_{\lambda}$. The white regions in this set are the Sierpinski holes.

In figure 1, we display the parameter plane (the $\lambda$-plane) for the family $F_{\lambda}$. The bounded white regions in this figure contain parameters for which $J\left(F_{\lambda}\right)$ is a Sierpinski curve; we call these regions Sierpinski holes.

By the above Theorem, any two Julia sets drawn from Sierpinski holes in parameter space are necessarily homeomorphic and hence these sets are identical from a topological point of view. But there are infinitely many of these holes in which the dynamics are different (i.e. the maps are not conjugate on their Julia sets). The basic reason for

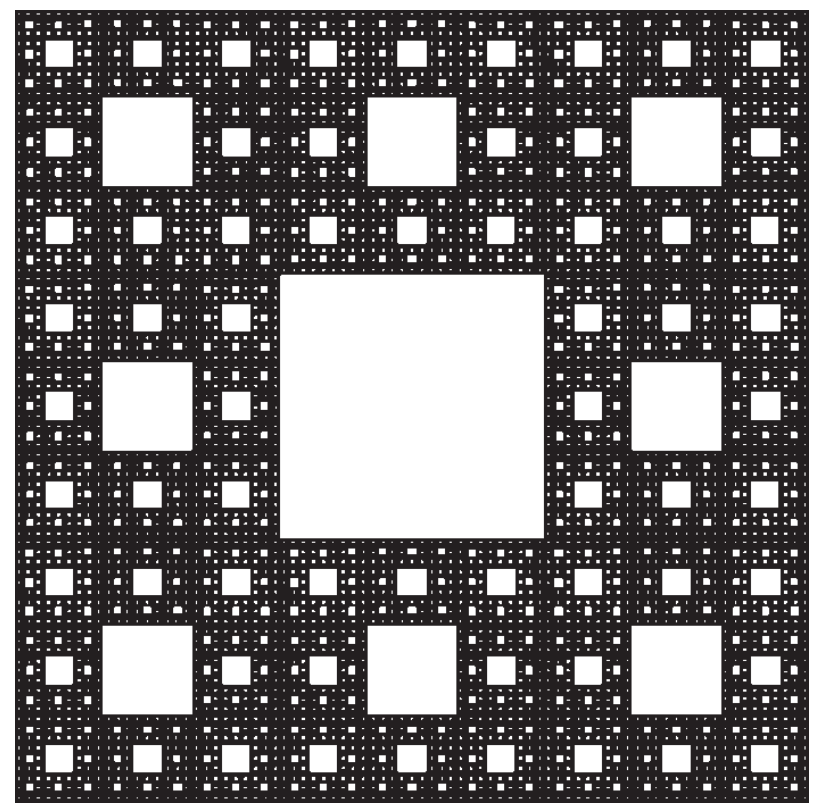

Figure 2. The Sierpinski carpet. 
the difference in the dynamical behavior is the following. In each Sierpinski hole, all of the critical points of $F_{\lambda}$ eventually escape to $\infty$, which is a superattracting fixed point. That is, the critical orbits eventually enter the immediate basin of attraction of $\infty$. If it takes a different number of iterations for the critical orbits to land in the immediate basin for different maps, then the corresponding maps cannot be conjugate as shown in [2]. Since there exist Sierpinski holes in which the critical orbits escape after exactly $k$ iterations for each $k \geq 3$, this explains why we have different dynamical behavior in certain of these regions (figure 1).

However, this fact does not provide a method for understanding the dynamics on the Sierpinski curve Julia sets of these maps. Our goal in this paper is to use symbolic dynamics to provide a complete description of this dynamical behavior on these sets. Rather than deal with the general case, we concentrate on a single example, namely when $\lambda=-1 / 16$. This $\lambda$ value is the center of the largest Sierpinski hole visible in the center of figure 1 . The Julia set of $F_{\lambda}$ when $\lambda=-1 / 16$ is displayed in figure 3 . This parameter value has dynamical behavior that is the simplest to understand, but it should be clear from the analysis below how to extend this analysis to other, more complicated maps drawn from different Sierpinski holes. Our main result is:

THEOREM There is a quotient space $\Sigma$ of the space of one-sided sequences on four symbols on which the shift map is conjugate to the dynamics of $F_{\lambda}$ on its Julia set.

\section{Elementary properties}

We begin by considering the basic mapping properties of maps in the family

$$
F_{\lambda}(z)=z^{2}+\frac{\lambda}{z^{2}}
$$

in the more general case, where $\lambda \in \mathbb{C}$ and $\lambda \neq 0$. For each such map, we have several symmetries: $F_{\lambda}(-z)=F_{\lambda}(z)$ and $F_{\lambda}(i z)=-F_{\lambda}(z)$ so that $F_{\lambda}^{2}(i z)=F_{\lambda}^{2}(z)$ for all $z \in \mathbb{C}$. Note that 0 is the only pole for each function in this family. The four critical points for $F_{\lambda}$ occur at $\lambda^{1 / 4}$, while the points $(-\lambda)^{1 / 4}$ are prepoles.

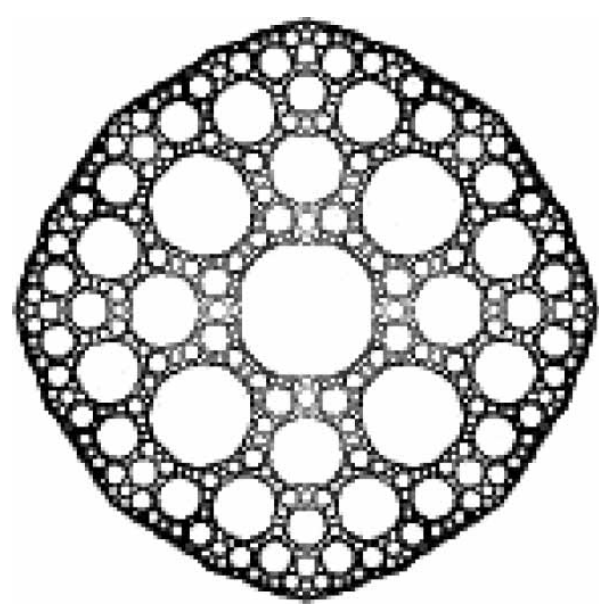

Figure 3. The Julia set for $F(z)=z^{2}-1 / 16 z^{2}$. 
The point at $\infty$ is a superattracting fixed point for $F_{\lambda}$. Let $B$ be the immediate basin of attraction of $\infty$ and denote by $\beta$ the boundary of $B$. The map $F_{\lambda}$ has degree 2 at $\infty$ and so $F_{\lambda}$ is conjugate to $z \mapsto z^{2}$ on $B$, at least in a neighborhood of $\infty$. The set $B$ is forward invariant under $F_{\lambda}$ in the sense that, if $z \in B$, then $F_{\lambda}^{n}(z) \in B$ for all $n \geq 0$. The same is true for $\beta$.

Let $J=J\left(F_{\lambda}\right)$ denote the Julia set of $F_{\lambda} . J$ is the set of points at which the family of iterates of $F_{\lambda}$ fails to be a normal family in the sense of Montel. Equivalently, $J$ is the closure of the set of repelling periodic points of $F_{\lambda}$ and it is also the boundary of the set of points whose orbits escape to $\infty$ (see [5]). As is the case for quadratic polynomials, $F_{\lambda}$ is chaotic on $J$.

We denote by $K=K\left(F_{\lambda}\right)$ the set of points whose orbit under $F_{\lambda}$ is bounded. $K$ is the filled Julia set of $F_{\lambda} . K$ is given by $\mathbb{C}-\cup F_{\lambda}^{-n}(B)$. Both $J$ and $K$ are completely invariant sets in the sense that if $z \in J$ (resp., $K$ ) then $F_{\lambda}^{n}(z) \in J$ (resp., $K$ ) for all $n \in \mathbb{Z}$. It is known that $J$ is the boundary of $K$ (see [9]). It is straightforward to check that each of the sets $B, \beta, J$ and $K$ are invariant under $z \mapsto i z$.

The following result is reminiscent of a similar result for quadratic polynomials. See [1] for a proof.

Proposition Suppose none of the critical points of $F_{\lambda}$ lie in $B$. Then $J\left(F_{\lambda}\right)$ and $K\left(F_{\lambda}\right)$ are connected sets. If one (and hence all) of the critical points lie in the immediate basin of $\infty$, then $J\left(F_{\lambda}\right)=K\left(F_{\lambda}\right)$ is a Cantor set and $F_{\lambda}\left|J\left(F_{\lambda}\right)\right|$ is conjugate to the one-sided shift map on four symbols.

The external white region in figure 1 contains all of the $\lambda$-values for which $J\left(F_{\lambda}\right)$ is a Cantor set. The complementary region in the parameter plane (including the Sierpinski holes) is the connectedness locus.

In the case where none of the critical points lie in $B$, it follows that $F_{\lambda}$ is two-to-one on $B$ and that $0 \notin B$. Let $T=F_{\lambda}^{-1}(B)-B$. Note that $T$ is a simply connected open set which is mapped in two-to-one fashion onto $B$, since $0 \in T$ is a pole of order two. $T$ is called the trap door since any orbit of $F_{\lambda}$ that enters $T$ immediately "falls through" it and enters the basin at $\infty$. As above, $T$ is also invariant under $z \mapsto i z$.

\section{A special case}

In this section, we restrict attention to

$$
F_{-1 / 16}(z)=z^{2}-\frac{1}{16 z^{2}},
$$

that is, the case where $\lambda=-1 / 16$. We denote $F_{-1 / 16}$ by $F$ for the remainder of this paper. In this section, we give two especially simple examples of how symbolic dynamics can be used to analyze the dynamics of $F$ on a pair of important invariant subsets of $J$. Later we use these two subsets as the cornerstone of the more complicated analysis of the dynamics on all of $J$.

The four critical points of $F$ lie at the points $\omega / 2$ where $\omega$ is a fourth root of -1 . The critical values are given by $\pm v= \pm i / 2$ and we have $F( \pm v)=0$. Thus the second iterate of each of the critical points lands on the pole at the origin; this is what makes the case $\lambda=-1 / 16$ special. There are prepoles at $\pm 1 / 2$ as well as at $\pm i / 2$.

We first investigate the dynamics of $F$ on $\mathbb{R}$. Note that $F$ preserves the real axis. The graph of $F$ on this axis shows that there is a pair of repelling fixed points in $\mathbb{R}$ (see figure 4). Let $p$ be 


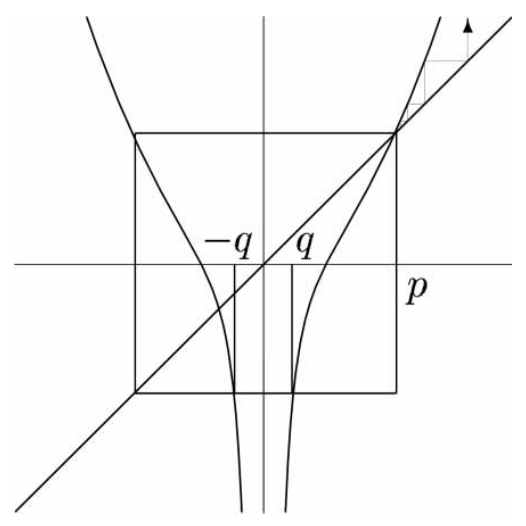

Figure 4. The graph of $F$ on the real line.

the fixed point in $\mathbb{R}^{+}$. The graph of $F$ also shows that the orbit of $x \in \mathbb{R}$ tends directly to $\infty$ if $|x|>p$, so $(p, \infty)$ and $(-\infty,-p)$ lie in $B$ and $\pm p \in \beta$.

Let $I$ denote the interval $[-p, p]$ and let $\Gamma$ be the set of points whose orbits remain in $I$ for all iterations. Let $\pm q \in I$ be the points for which $F( \pm q)=-p$ so that $F^{2}( \pm q)=p$. If $x \in(-q, q)$ then $F(x)<-p$ and $F^{2}(x)>p$. Hence $F^{n}(x) \rightarrow \infty$ for all $x \in(-q, q)$. This interval is the trap door in $\mathbb{R}$; any orbit in $I$ that enters this open interval falls through the trap door and then tends to $\infty$.

Proposition The set of points $\Gamma$ whose orbits remain in $[-p, p]$ for all iterations is a Cantor set, and $F$ is conjugate to the one-sided shift map on two symbols on this set. Moreover $\Gamma \subset J(F)$.

Proof. An easy computation shows that $\left|F^{\prime}(x)\right|$ has a minimum in $\mathbb{R}$ at $\pm 3^{1 / 4} / 2$, and that the minimum value of $\left|F^{\prime}(x)\right|$ is $3^{1 / 4}+3^{-3 / 4} \approx 1.75$. Hence, $F$ is expanding on $[-p, p]$. Let $I_{a}=[q, p]$ and $I_{b}=[-p,-q]$. Let $\Sigma_{2}$ be the sequence space on the two symbols $a$ and $b$, and let $S: \Gamma \rightarrow \Sigma_{2}$ be the itinerary map that assigns the sequence $\left(s_{0} s_{1} s_{2} \ldots\right)$ to $x \in \Gamma$ where $s_{j}=a$ if $F^{j}(x) \in I_{a}$ or $S_{j}=b$ otherwise. Then standard arguments show that $S$ is a homeomorphism that conjugates $F \mid \Gamma$ to the shift map on $\Sigma_{2}$. Therefore, $\Gamma$ is a Cantor set.

Since the orbits of points in $\Gamma$ are bounded, it follows that $\Gamma$ is contained in the filled Julia set of $F$. The set of points in $\Gamma$ whose orbits eventually land on $p$ are dense in $\Gamma$, and these points lie in the boundary of the set of escaping points. Hence, the closure of this set of points, namely $\Gamma$, lies in $J(F)$.

The preimage of $\mathbb{R}$ under $F_{\lambda}$ consists of the real and imaginary axes; each of these axes is mapped two-to-one over $\mathbb{R}$. Hence there is a Cantor set on the imaginary axis that is mapped in two-to-one fashion onto $\Gamma$ in $\mathbb{R}$, and all other points on the imaginary axis lie in $B$.

The preimage of the imaginary axis consists of two sets: the four rays $\theta= \pm \pi / 4, \pm 3 \pi / 4$ and the circle of radius $r=1 / 2$ centered at the origin. We call this circle the critical circle. Note that the four rays meet the critical circle at the four critical points of $F$. A point on the critical circle given by $r=(1 / 2) e^{i \theta}$ is mapped to points of the form $(i / 2) \sin (2 \theta)$ on the imaginary axis. Therefore, the critical circle is mapped in four-to-one fashion over the 
interval $[-1 / 2,1 / 2]$ on the imaginary axis (except at the endpoints). Each of the four rays is mapped in two-to-one fashion over either $[1 / 2, \infty)$ or $(-\infty,-1 / 2]$ on the imaginary axis.

We now investigate the behavior of $F_{\lambda}$ near $\infty$.

Proposition The boundary of the basin of attraction of $\infty$ is an invariant simple closed curve $\beta$ on which $F$ is conjugate to the map $z \rightarrow z^{2}$.

Proof. Let $W$ denote the annulus given by $3 / 4 \leq|z| \leq 2$. We claim that $F$ is an expanding, two-to-one covering map on $W$. To see this, first consider the circle $r=(3 / 4) e^{i \theta}$. For $z$ on this circle, we have

$$
|F(z)|=\left|\frac{9}{16} e^{2 i \theta}-\frac{1}{9} e^{-2 i \theta}\right| \leq \frac{9}{16}+\frac{1}{9}<\frac{3}{4} .
$$

Hence, $F$ maps this circle strictly inside itself. If $|z| \geq 2$, then we have

$$
|F(z)| \geq|z|^{2}-\frac{1}{16|z|^{2}} \geq|z|^{2}-\frac{1}{64}>1.5|z| .
$$

therefore, $F$ maps the circle $|z|=2$ strictly outside itself and we have

$$
\left|F^{n}(z)\right| \geq(1.5)^{n}|z|
$$

for all $n \geq 1$ and $|z| \geq 2$. Therefore, the entire region $|z| \geq 2$ lies in $B$.

If $|z| \geq 3 / 4$, we also have that

$$
\left|F^{\prime}(z)\right| \geq 2|z|-\frac{1}{8|z|^{3}} \geq \frac{3}{2}-\frac{8}{27}>1,
$$

so it follows that $F$ is expanding on the annulus $W$. Since the critical points lie inside the circle $|z|=3 / 4$, it follows that $F_{\lambda}$ is a two-to-one covering map on $W$, and $F_{\lambda}(W) \supset W$. Standard arguments then show that the set of points whose orbits remain for all iterations inside $W$ is a simple closed curve, and $F_{\lambda}$ is conjugate to $z^{2}$ on this curve. Moreover, all points outside this curve lie in $B$, and so this invariant curve is $\beta$.

As a consequence of this result, the boundary of the trap door is also a simple closed curve in $\mathbb{C}$, as are all other preimages of $\beta$. We denote the boundary of the trap door by $\tau$. Note that $F$ maps $\tau$ onto $\beta$ in two-to-one fashion and that $\tau$ is disjoint from $\beta$. By the Proposition, we also have the fact that $K(F)$ is the set of all points whose orbits remain for all time on or inside $\beta$. We also remark that a similar result holds for other $\lambda$-values for which the critical orbit eventually enters $T$; the proof is more complicated as it involves quasiconformal surgery and the Measurable Riemann Mapping Theorem (see [3,4]).

With an eye toward our discussion of the full symbolic dynamics on the Julia set, we introduce the usual coding of orbits on $\beta$. Let $\Sigma_{2}^{\prime}$ be the sequence space on the two symbols 0 and 1 (not $a$ and $b$, as before). In $\Sigma_{2}^{\prime}$ we identify two sequences that begin with the same finite string of digits and end in either all zeroes or ones. That is, if $s=\left(s_{0} \ldots s_{n} \overline{0}\right)$ and $t=\left(s_{0} \ldots s_{n} \overline{1}\right)$, then we identify these two points in $\Sigma_{2}^{\prime}$. Let $\tilde{\Sigma}_{2}^{\prime}$ denote the corresponding quotient space.

There is a natural conjugacy between the dynamics of $F$ on $\beta$ and the one-sided shift map on $\tilde{\Sigma}_{2}^{\prime}$ determined as follows. Let $K_{0}$ denote the portion of $\beta$ lying on or above the real axis, and let $K_{1}$ denote that portion lying on or below this axis. Note that, by our 
previous work, we know that $\beta$ meets the real axis only at the points $\pm p$. We then associate an itinerary $S(z)$ in $\tilde{\Sigma}_{2}^{\prime}$ to each $z \in \beta$ by recording how the orbit of $z$ visits either $K_{0}$ or $K_{1}$ exactly as in the case of the dynamics on the real line. Since $K_{0} \cap K_{1}=\{ \pm p\}$, it follows immediately that this assignment respects the identifications in $\tilde{\Sigma}_{2}^{\prime}$ and yields a conjugacy.

Proposition The itinerary map $S$ gives a conjugacy between $F \mid \beta$ and the one-sided shift map on the quotient space $\tilde{\Sigma}_{2}^{\prime}$.

\section{Cantor necklaces}

One of the principal objects contained in the Julia set of $F$ is a Cantor necklace. To define this set, let $\Lambda$ denote the Cantor middle thirds set in the unit interval $[0,1]$. We regard this interval as a subset of the real axis in the plane. For each open interval of length $1 / 3^{n}$ removed from the unit interval in the construction of $\Lambda$, we replace this interval by a circle of diameter $1 / 3^{n}$ centered at the midpoint of the removed interval. Thus this circle meets the Cantor set at the two endpoints of the removed interval. We call the resulting set the Cantor middle-thirds necklace (see figure 5). Any set homeomorphic to the Cantor middle-thirds necklace is called a Cantor necklace.

There is a natural invariant Cantor necklace contained in $J(F)$. Recall that the boundary $\tau$ of the trap door $T$ is mapped in two-to-one fashion onto the boundary $\beta$ of $B$. Moreover, $\tau$ and $\beta$ are disjoint and $\tau$ meets $\mathbb{R}$ only at the two points $\pm q$. There are four preimages of $T$ in $\mathbb{C}$, but only two of them meet $\mathbb{R}$. These two preimages of $T$ meet the real axis in the two open intervals that were removed at the first stage of the construction of the invariant Cantor set $\Gamma$ described in the previous section. Hence, their boundaries are simple closed curves that meet $\Gamma$ in two points that are the preimages of $\pm q$. Since $\beta$ and $\tau$ are disjoint, it follows that these two curves are also disjoint from $\beta$ and $\tau$. Continuing in this fashion, at the $n$th stage, we replace the $n$th intervals removed in the Cantor set construction with the corresponding $n$th preimage of $\tau$. The resulting set is then a Cantor necklace $\mathcal{N}$, and $\mathcal{N} \subset J(F)$ since every point in this set lies in the closure of $\cup F^{-n}(B)$ (see [3]).

Now consider $\beta \cup \mathcal{N}$. This set is invariant under $F$. Any point in the Cantor set portion $\Gamma$ of $\mathcal{N}$ has orbit that remains in $\Gamma$, whereas any other point in $\mathcal{N}$ eventually maps to $\beta$, where the orbit is then trapped. We could use symbolic dynamics to expand our previous symbolic description to this set, but we will instead take a wider viewpoint and use this set as the skeleton of a larger symbolic description.

\section{Symbolic dynamics on the Julia set}

In this section, we give a symbolic description of the itinerary of each point in the Julia set of $F$. Let $A$ be the closed annulus bounded by $\beta$ and $\tau$. All points in $J(F)$ are contained in $A$. Indeed, $J(F)$ is $A$ minus all of the preimages of $T$. We divide $A$ into four overlapping closed



Figure 5. The Cantor middle-thirds necklace. 


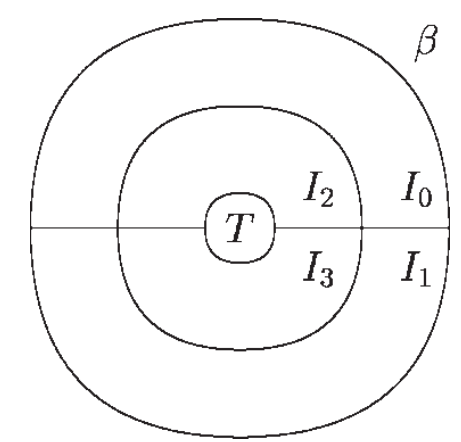

Figure 6. The regions $I_{j}$ for $j=0,1,2,3$.

sets $I_{0}, I_{1}, I_{2}$ and $I_{3}$, each of which is a semi-annulus. $I_{0}$ and $I_{2}$ lie the upper half plane while $I_{1}$ and $I_{3}$ lie in the lower half plane. $I_{0}$ and $I_{1}$ are bounded by portions of $\beta$, the critical circle, and $\mathbb{R}$, while $I_{2}$ and $I_{3}$ are bounded by portions of $\tau$, the critical circle, and $\mathbb{R}$ (see figure 6 ).

Note that $F$ maps each $I_{j}$ onto all of $\mathbb{C}-B$. For example, the boundary of $I_{0}$ is mapped by $F$ as follows:

1. $I_{0} \cap \beta$ is mapped one-to-one to all of $\beta$ (two-to-one to $p$ );

2. $I_{0} \cap \mathbb{R}^{+}$is mapped one-to-one to $[0, p] \subset \mathbb{R}^{+}$;

3. $I_{0} \cap \mathbb{R}^{-}$is mapped one-to-one to $[-p, 0] \subset \mathbb{R}^{-}$;

4. the portion of the critical circle bounding $I_{0}$ is mapped two-to-one to the interval $[-v, v]$ on the imaginary axis.

Let $\Sigma$ denote the set of one-sided sequences on the four symbols $0,1,2$ and 3 . We assign a sequence $S(z)=\left(s_{0} s_{1} s_{2} \ldots\right) \in \Sigma$ to each $z \in J(F)$ in the usual fashion so that the entry $S_{j}=k$ if and only if $F^{j}(z) \in I_{k} . S(z)$ is the itinerary of $z$. Since $F\left(I_{j}\right) \supset \mathbb{C}-B$ for each $j$, it follows easily that there is at least one point in $\mathbb{C}-B$ associated to each sequence in $\Sigma$. These points have bounded orbits and so must lie in $J$. So $S$ gives a map from $J(F)$ onto $\Sigma$.

In order for $S$ to capture the precise dynamics of $F$ on the Julia set, we need to modify this picture in several ways. First, certain points in $J(F)$ correspond to two different sequences since the $I_{j}$ overlap, so we must first make a number of identifications in $\Sigma$, much as we did earlier on $\beta$. Second, $F$ will not be conjugate to the shift map on this quotient space, but rather to a "quotient" of the shift map.

We first modify $\Sigma$ to take into account the identifications necessary along $\beta$. As in section 3 , we identify any pair of sequences in $\Sigma$ of the form $\left(s_{0} s_{1} \ldots s_{n} \overline{0}\right)$ and $\left(s_{0} s_{1} \ldots s_{n} \overline{1}\right)$. Let $\Sigma^{1}$ denote the resulting quotient space. Then we have:

Proposition Let $s=\left(s_{0} s_{1} s_{2} \ldots\right) \in \Sigma^{1}$ be a sequence consisting of only $0 s$ or $1 s$. Then there is a unique point in $J(F)$ whose itinerary is $s$ and this point lies in $\beta$.

Proof. Let $I_{s_{0} s_{1} \ldots s_{n}}$ denote the set of points whose itinerary begins $s_{0} s_{1} \ldots s_{n}$. If each of the $s_{j}$ is either 0 or 1 , then $I_{s_{0} s_{1} \ldots s_{n}}$ is a closed, simply connected set that is properly contained in $I_{s_{0} s_{1} \ldots s_{n-1}}$ and $F^{n}$ maps $I_{s_{0} s_{1} \ldots s_{n}}$ in one-to-one fashion onto $I_{s_{n}}$. Note that each $I_{s_{0} s_{1} \ldots s_{n}}$ intersects $\beta$ in an arc. Using the appropriate branches of the inverse of $F$, we have that $F^{-n}$ is a contraction in the Poincaré metric on $I_{s_{n}}$ taking $I_{s_{n}}$ onto $I_{s_{0} s_{1} \ldots s_{n}}$. Hence the intersection of these sets as $n \rightarrow \infty$ is a unique point which necessarily lies in $\beta$, and this point has the itinerary $s$. 
The description of the further identifications that must be made in $\Sigma^{1}$ is more complicated. The reason for this is that the sets $I_{s_{0} s_{1} \ldots s_{n}}$ are not always simply connected if the $s_{j}$ involve $2 \mathrm{~s}$ or $3 \mathrm{~s}$. For example, the interior of the set $I_{02}$ consists of two disjoint components whose boundaries meet at a single critical point.

\subsection{Symbolic dynamics on the real line}

To describe the further identifications in $\Sigma^{1}$, we begin on the real axis. Note that our previous symbolic description of points in $J(F) \cap \mathbb{R}$ (using the symbols $a$ and $b$ ) is no longer valid. Now we have four symbols describing such points and each point in $J(F) \cap \mathbb{R}$ will have two distinct symbolic representations.

Recall that $\mathcal{N}$ denotes the Cantor necklace lying along $\mathbb{R}$ and that $\Gamma$ denotes the Cantor set given by $\mathcal{N} \cap \mathbb{R}$. We have $F(\mathcal{N})=\mathcal{N} \cup \beta$. If we remove the set of points in $\mathcal{N}$ whose images do not lie in $\mathcal{N}$, then we are left with a pair of Cantor subnecklaces, one to the left of the trap door $T$ (extending from $-p$ to $-q$ along $\mathbb{R}^{-}$) and one to the right of $T$ (extending from $q$ to $p$ along $\mathbb{R}^{+}$). Indeed, we simply remove the upper and lower open semicircles on the boundary of $T$ (i.e. $\tau-\{ \pm q\}$ ) from $\mathcal{N}$ to produce these subnecklaces.

The preimage in $\mathbb{R}$ of this pair of subnecklaces then consists of four Cantor subnecklaces, and then the preimage in $\mathbb{R}$ of these subnecklaces consists of eight subnecklaces. Call this set of eight subnecklaces $\mathcal{N}^{\prime}$.

Points that lie in the upper or lower portions of a subnecklace in $\mathcal{N}^{\prime}$ can be distinguished using a pair of symbols. Recall that $I_{\alpha \beta}$ denotes the set of points in $J$ whose itinerary begins with the string $\alpha \beta$. Each of the eight subnecklaces in $\mathcal{N}^{\prime}$ is associated to a pair of distinct $I_{\alpha \beta}$; one of the $I_{\alpha \beta}$ contains all points in the upper half of a subnecklace in $\mathcal{N}^{\prime}$, the other to the bottom half. Using the dynamics of $F$ on $\mathbb{R}$, we see that the upper pieces of the subnecklaces in $\mathcal{N}^{\prime}$ are contained from left to right in

$$
I_{01}, I_{03}, I_{23}, I_{21}, I_{20}, I_{22}, I_{02}, I_{00} \text {. }
$$

The bottom pieces are contained from left to right in

See figure 7.

$$
I_{10}, I_{12}, I_{32}, I_{30}, I_{31}, I_{33}, I_{13}, I_{11} \text {. }
$$

Applying $F$, we then see that, for example,

$$
F\left(I_{00}\right) \supset I_{00} \cup I_{02}
$$

and

$$
F\left(I_{23}\right) \supset I_{32} \cup I_{30} \text {. }
$$

This implies that points in $I_{00}$ have itineraries that begin 000 or 002 while points in $I_{23}$ have itineraries that begin 230 or 232 . We write this more succinctly as

$$
00 \rightarrow 00\{0,2\} \quad \text { and } 23 \rightarrow 23\{0,2\} .
$$

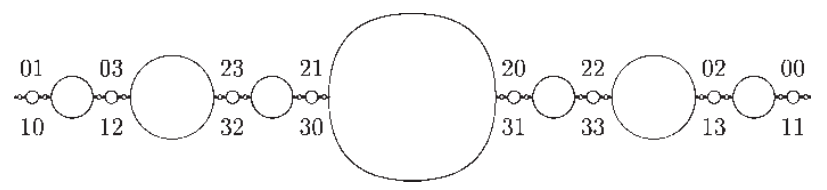

Figure 7. Symbolic dynamics on the Cantor necklace. 
A straightforward computation shows that we have, in order,

$$
\begin{array}{llll}
00 \rightarrow 00\{0,2\}, & 02 \rightarrow 02\{0,2\}, & 22 \rightarrow 22\{1,3\}, & 20 \rightarrow 20\{1,3\}, \\
21 \rightarrow 21\{0,2\}, & 23 \rightarrow 23\{0,2\}, & 03 \rightarrow 03\{1,3\}, & 01 \rightarrow 01\{1,3\}, \\
11 \rightarrow 11\{1,3\}, & 13 \rightarrow 13\{1,3\}, & 33 \rightarrow 33\{0,2\}, & 31 \rightarrow 31\{0,2\}, \\
30 \rightarrow 30\{1,3\}, & 32 \rightarrow 32\{1,3\}, & 12 \rightarrow 12\{0,2\}, & 10 \rightarrow 10\{0,2\},
\end{array}
$$

Note that, in this recipe, if an even digit is preceded by a 0 or 1 , then the following digit must also be even. If the even digit is preceded by a 2 or 3 , the following digit must be odd. Similarly, an odd digit preceded by 0 or 1 is followed by an odd digit while it is followed by an even digit if preceded by a 2 or 3 . More succinctly, we have:

Proposition (The Criterion for Real Itineraries.) Suppose $s=\left(s_{0} s_{1} s_{2} \ldots\right)$ is a sequence that corresponds to a point in $J \cap \mathbb{R}$. Then for each $j, s_{j}$ satisfies

1. If $s_{j}=0$ or 1 , then $s_{j+1}$ and $s_{j+2}$ have the same parity;

2. If $s_{j}=2$ or 3 , then $s_{j+1}$ and $s_{j+2}$ have different parities.

Conversely, any sequence that satisfies this rule for each $s_{j}$ corresponds to a unique point in $J \cap \mathbb{R}$.

We say that a sequence in $\Sigma^{1}$ corresponds to a real itinerary if it obeys this criterion for all $j$. Using the criterion for real itineraries, we can now "identify" all of the sequences corresponding to points in $J \cap \mathbb{R}$. Let $R: \Sigma^{1} \rightarrow \Sigma^{1}$ be the map that exchanges 0's and 1's or 2's and 3's in each sequence in $\Sigma^{1}$. Then we have that, if $s \in \Sigma^{1}$ corresponds to a point in $J \cap \mathbb{R}$, then $R(s)$ also corresponds to such a point and, moreover, these two points are the same. Therefore, we identify any two such sequences in $\Sigma^{1}$. Note that, under this identification, the sequences $(\overline{0})$ and $(\overline{1})$ are identified, as are $(0 \overline{1})$ and $(1 \overline{0})$. These represent the two points where this new identification coincides with the previous identification.

Now suppose we have a sequence in $\Sigma^{1}$ that does not correspond to a real itinerary. Then there are two possibilities:

1. Either there are only finitely many $s_{j}$ for which the criterion for a real itinerary fails, or

2. There are infinitely many $s_{j}$ for which this criterion fails.

In the first case, such a sequence must be of the form

$$
\left(s_{0} \ldots s_{n} t_{n+1} t_{n+2} \ldots\right)
$$

where $n$ is the largest digit for which the criterion for real itineraries fails. We say that $n$ is the real itinerary marker. If the real itinerary marker is 0 , then any point with such an itinerary lies along the imaginary axis. Any such point corresponds to two distinct itineraries having the form $(j,(s))$ and $(j, R(s))$ where $j=0,1,2$, or 3 and $s$ is a sequence that corresponds to a real itinerary. Therefore, we identify these two sequences in $\Sigma^{1}$ as well.

If the real itinerary marker is 1 , then the situation is different. In this case, the corresponding points in $J$ lie either on the critical circle or on the four straight rays connecting the origin to $\infty$ and passing through one of the critical points. The points lying on the critical circle are mapped to the portion of the imaginary axis in $I_{2} \cup I_{3}$ while the other 
points are mapped to the portion in $I_{0} \cup I_{1}$. The former case presents a problem since points on the critical circle lie on the boundaries between two of the $I_{j}$; we deal with this more complicated case in the next section. In the latter case, the itinerary of such a point is either $\left(s_{0}, j,(s)\right)$ or $\left(s_{0}, j, R(s)\right)$ where $j=0$ or $j=1$ and $s$ is a sequence that corresponds to a real itinerary. Hence, we identify two such sequences as above.

Finally, if the real itinerary marker is $j \geq 2$ and $s_{j}=0$ or 1 , we have a similar pair of sequences that correspond to the same point. Again these two sequences are identified. With all of these identifications, we now have a quotient space of $\Sigma^{1}$ that we call $\Sigma^{2}$.

\subsection{Symbolic dynamics on the critical circle}

The only other points where the $I_{j}$ intersect lie along the critical circle, so we now describe the identifications that these intersections cause in $\Sigma^{2}$. Sequences that correspond to points on the critical circle have the form $\left(s_{0}, j,(s)\right)$ where $j=2$ or $j=3$ and $s$ corresponds to a real itinerary.

Let $C$ denote the critical circle. Recall that $F$ maps $C$ four-to-one onto the portion of the imaginary axis lying between the two critical values $\pm v$. One of these critical values is located at the intersection of $I_{0}$ and $I_{2}$ on the positive imaginary axis; the other is located at the intersection of $I_{1}$ and $I_{3}$ on the negative imaginary axis.

Recall also that $F$ maps the portion of the imaginary axis lying in $I_{2}$ in one-to-one fashion onto the interval $[0, p]$ where we recall that $p$ is the fixed point in $\mathbb{R}^{+}$and $q$ is the preimage of $-p$ lying in $\mathbb{R}^{+}$. So there is a Cantor subnecklace along this portion of the imaginary axis that is mapped onto the portion of the Cantor necklace $\mathcal{N}$ along the interval $[q, p]$. We call this subnecklace $U$. There is a similar Cantor subnecklace $V=-U$ in $I_{3}$ that is also mapped homeomorphically onto the same portion of $\mathcal{N}$ (see figure 8).

For simplicity, in our figures we will henceforth represent the subnecklaces $U$ and $V$ by lines (see figure 9).

$U$ can be divided into two equal sized subnecklaces which we will denote $U_{\text {in }}$ and $U_{\text {out }}$ with $U_{\text {out }}$ lying above $U_{\text {in }}$ on $i \mathbb{R}$. We create this division by removing the set in $U$ that is mapped onto $\tau-\{ \pm q\}$ in two iterations. Likewise, let $V_{\text {in }}=-U_{\text {in }}$ and $V_{\text {out }}=-U_{\text {out }}$ (see figure 10). Any point in the Cantor set portion of these subnecklaces lying in $I_{j}$ has itinerary $\left(j s_{0} s_{1} \ldots\right)$ where each entry in the sequence $\left(s_{0} s_{1} s_{2} \ldots\right)$

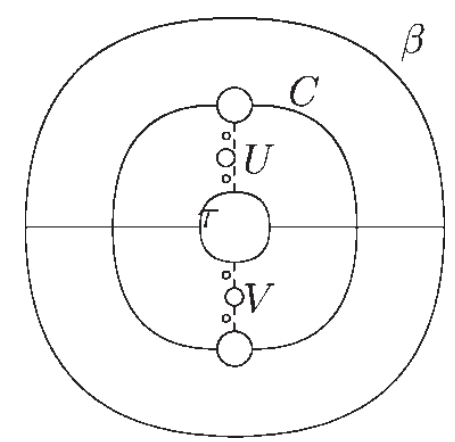

Figure 8. The Cantor subnecklaces $U$ and $V$. 


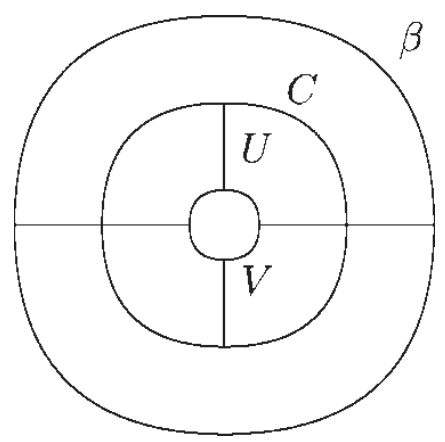

Figure 9. The Cantor subnecklaces $U$ and $V$ represented by lines.

satisfies the criterion for a real itinerary but the initial digit $j$ does not, so the real itinerary marker is 0 .

For the identifications that occurred along the real line we needed distinct notations for the upper and lower pieces of the Cantor necklace along $\mathbb{R}$. Similarly, we will speak of the left and right pieces of $U$ and $V$. Our convention will be that points in the right pieces have nonnegative real parts while points in the left pieces have nonpositive real parts. For example, we will denote the left piece of $U_{\text {out }}$ by $U_{(\mathrm{out}, \mathrm{L})}$ and the right piece by $U_{(\mathrm{out}, \mathrm{R})}$. Since $U \subset I_{2}$ and $V \subset I_{3}$, it follows that any point in $U$ has itinerary that begins with 2 while any point in $V$ has itinerary that begins with 3 . It is a matter of computation to see that $F\left(U_{\text {(out,R) }}\right) \subset I_{2}$ and that $F^{(2)}\left(U_{(\text {out }, \mathrm{R})}\right)$ is contained is $I_{0}$ or $I_{2}$. Hence, itineraries of points in $U_{\text {(out,R) }}$ must begin with 220 or 222 . We will write this as

$$
U_{(\text {out }, \mathrm{R})} \rightarrow 22\{0,2\} .
$$

Adopting this notation, we compute immediately that

$$
\begin{array}{cccc}
U_{(\text {out }, \mathrm{R})} \rightarrow 22\{0,2\}, & U_{(\mathrm{out}, \mathrm{L})} \rightarrow 23\{1,3\}, & U_{(\mathrm{in}, \mathrm{L})} \rightarrow 21\{1,3\}, & U_{(\mathrm{in}, \mathrm{R})} \rightarrow 20\{0,2\} \\
V_{(\mathrm{in}, \mathrm{R})} \rightarrow 31\{1,3\}, & V_{(\mathrm{out}, \mathrm{R})} \rightarrow 33\{1,3\}, & V_{(\mathrm{in}, \mathrm{L})} \rightarrow 30\{0,2\}, & V_{(\mathrm{out}, \mathrm{L})} \rightarrow 32\{0,2\} .
\end{array}
$$

We turn now to the identifications in $\Sigma^{2}$ corresponding to points in $C$. Recall that $C$ is mapped four-to-one onto the interval $[-v, v]$ on the imaginary axis. Consider the preimage of the Cantor necklace $U$ along the critical circle. This preimage consists of four Cantor necklaces, two in each of the first and third quadrants. Each of these preimages is mapped

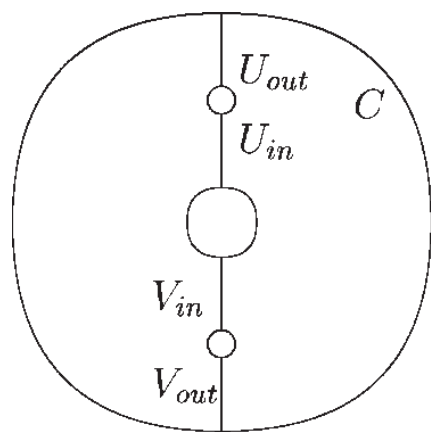

Figure 10. Location of $U_{\text {in }}$ and $U_{\text {out }}$ within $C$. 


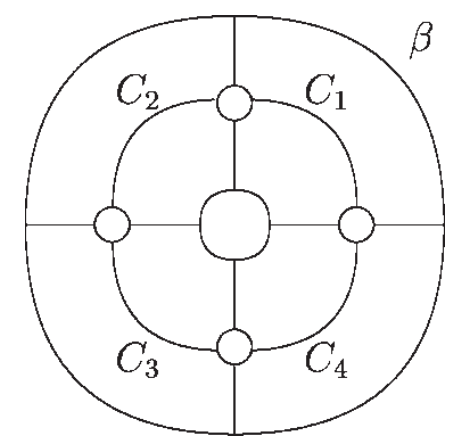

Figure 11. The location of the Cantor subnecklaces $C_{i}$ for $i=1,2,3,4$.

one-to-one onto $U$. Similarly, the necklace $V$ has four preimage necklaces, two each in the second and fourth quadrants. Let $C_{1}$ denote the two preimages of $U$ lying in the first quadrant. Continuing counterclockwise from $C_{1}$, we label the other preimages of $U$ or $V$ by $C_{2}, C_{3}$ and $C_{4}$ (see figure 11).

Note that $C_{1}$ and $C_{2}$ lie along the critical circle separating $I_{0}$ from $I_{2}$ while $C_{3}$ and $C_{4}$ lie on the critical circle separating $I_{1}$ from $I_{3}$. We next break each of the $C_{j}$ into four equal sized subnecklaces by removing the middle preimage of $\tau$ from each. Equivalently, we consider the preimages of the smaller subnecklaces $U_{\text {in }}, U_{\text {out }}, V_{\text {in }}$, and $V_{\text {out }}$. This yields sixteen Cantor subnecklaces lying along the critical circle, each of which is mapped to the one of the subnecklaces on the imaginary axis. Each of these sixteen subnecklaces has two pieces; one outside $C$ and one inside $C$. The outside piece of $C_{1}$ that lies in $I_{0}$ and meets the preimage of $\tau$ along $\mathbb{R}^{+}$is mapped to $U_{(\mathrm{in}, \mathrm{R})}$. Starting at this piece and continuing counterclockwise along the critical circle, the outer pieces of the sixteen Cantor subnecklaces lying in $I_{0} \cup I_{1}$ are mapped to

$$
\begin{array}{llllllll}
U_{(\text {in }, \mathrm{R})}, & U_{(\text {out }, \mathrm{R})}, & U_{(\text {out }, \mathrm{L})}, & U_{(\text {in }, \mathrm{L})}, & V_{(\text {in }, \mathrm{L})}, & V_{(\text {out }, \mathrm{L})}, & V_{(\text {out }, \mathrm{R})}, & V_{(\text {in }, \mathrm{R})}, \\
U_{(\text {in }, \mathrm{R})}, & U_{(\text {out }, \mathrm{R})}, & U_{(\text {out }, \mathrm{L})}, & U_{(\text {in }, \mathrm{L})}, & V_{(\text {in }, \mathrm{L})}, & V_{(\text {out }, \mathrm{L})}, & V_{(\text {out }, \mathrm{R})}, & V_{(\mathrm{in}, \mathrm{R})},
\end{array}
$$

respectively. Figure 12 shows the pieces of $C_{1}$ in $I_{0}$ (as well as the pieces in $I_{2}$ ) marked by where they are mapped via $F$. The first eight of these pieces of necklaces live in $I_{0}$ while the

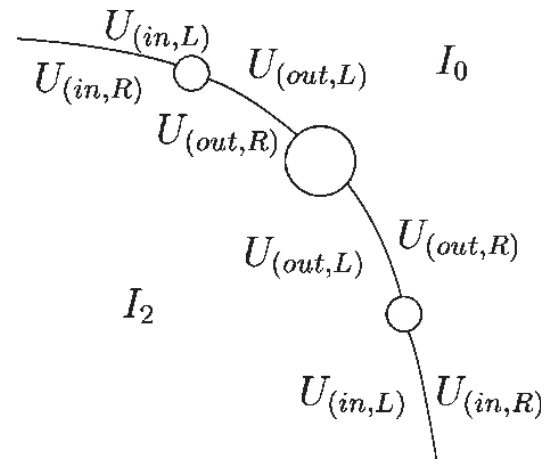

Figure 12. The locations of the eight pieces of $C_{1}$ marked via their images. 
second eight live in $I_{1}$, giving us itineraries that begin, in the above order, with

$$
\begin{array}{llll}
020\{0,2\}, & 022\{0,2\}, & 023\{1,3\}, & 021\{1,3\}, \\
030\{0,2\}, & 032\{0,2\}, & 033\{1,3\}, & 031\{1,3\}, \\
120\{0,2\}, & 122\{0,2\}, & 123\{1,3\}, & 121\{1,3\}, \\
130\{0,2\}, & 132\{0,2\}, & 133\{1,3\}, & 131\{1,3\} .
\end{array}
$$

Now, starting at the inner piece of $C_{1}$ that lies in $I_{2}$ and intersects the preimage of $\tau$ on the positive real axis and continuing counterclockwise along the critical circle we see that the inner pieces of the sixteen Cantor subnecklaces lying in $I_{2} \cup I_{3}$ are mapped to

$$
\begin{array}{llllllll}
U_{(\text {in }, \mathrm{L})}, & U_{(\text {out }, \mathrm{L})}, & U_{(\text {out }, \mathrm{R})}, & U_{(\mathrm{in}, \mathrm{R})}, & V_{(\mathrm{in}, \mathrm{R})}, & V_{(\text {out }, \mathrm{R})}, & V_{(\text {out }, \mathrm{L})}, & V_{(\mathrm{in}, \mathrm{L})}, \\
U_{(\mathrm{in}, \mathrm{L})}, & U_{(\text {out }, \mathrm{L})}, & U_{(\text {out }, \mathrm{R})}, & U_{(\text {in }, \mathrm{R})}, & V_{(\text {in }, \mathrm{R})}, & V_{(\text {out }, \mathrm{R})}, & V_{(\text {out }, \mathrm{L})}, & V_{(\mathrm{in}, \mathrm{L})},
\end{array}
$$

respectively. The itineraries of points in these sets begin

$$
\begin{array}{llll}
221\{1,3\}, & 223\{1,3\}, & 222\{0,2\}, & 220\{0,2\}, \\
231\{1,3\}, & 233\{1,3\}, & 232\{0,2\}, & 230\{0,2\}, \\
321\{1,3\}, & 323\{1,3\}, & 322\{0,2\}, & 320\{0,2\}, \\
331\{1,3\}, & 333\{1,3\}, & 332\{0,2\}, & 330\{0,2\} .
\end{array}
$$

Note that the second entry for all 32 of these itineraries is either a 2 or a 3 . This is because the critical circle is mapped onto the portion of the imaginary axis between the boundary of the trap door and the critical value, and this region is in $I_{2} \cup I_{3}$.

With this information, we can now identify the itineraries of points lying on the critical circle.

Proposition (Criterion for a Critical Circle Itinerary.) Any point in $J(F) \cap C$ has itinerary of the form $\left(s_{0} s_{1} s_{2} \ldots\right)$ where the real itinerary marker is 1 and either $s_{1}=2$ or $s_{1}=3$. Conversely, any itinerary that satisfies these conditions corresponds to a point in $J(F) \cap C$.

Finally, let $s=\left(s_{0} s_{1} s_{2} \ldots\right)$ be a sequence in $\Sigma^{2}$ corresponding to a point in $J \cap C$ and let $H: \Sigma^{2} \rightarrow \Sigma^{2}$ denote the map that changes $s_{0}$ and $s_{j}$ for each $j \geq 2$ as follows:

1. $0 \mathrm{~s}$ are interchanged with $2 \mathrm{~s}$ and $1 \mathrm{~s}$ are interchanged with $3 \mathrm{~s}$ in $s_{0}$,

2. $0 \mathrm{~s}$ are interchanged with $1 \mathrm{~s}$ and $2 \mathrm{~s}$ are interchanged with $3 \mathrm{~s}$ in $s_{j}$ if $j \geq 2$.

If $s \in \Sigma^{2}$ corresponds to a point in $J \cap C$ then $H(s)$ also corresponds to such a point and, moreover, the points corresponding to $s$ and $H(s)$ are the same. Hence, we identify these sequences in $\Sigma^{2}$. Similarly any pair of sequences whose first $n$ entries are the same and whose tail is one of these two identified sequences should also be identified in $\Sigma^{2}$. This then gives a new quotient space $\Sigma^{3}$.

We remark that there are two points in $C_{1}$ that are mapped to the same point on the imaginary axis. If one of these points corresponds to the identified sequences $(02 s)$ and $(22 R(s))$, then the other point corresponds to the pair of identified sequences $(22 s)$ and 
$(02 R(s))$. So the sequences corresponding to the images of these points are $(2 s)$ and $(2 R(s))$, both of which have already been identified in $\Sigma^{3}$.

Note also that the sequences that have been identified to form the quotient space $\Sigma^{3}$ correspond to points in $J(F)$ whose orbits eventually land in the invariant Cantor set in $\mathbb{R}$.

\subsection{Symbolic dynamics}

Given all of our work constructing the space $\Sigma^{3}$, we can now prove:

THEOREM The map $F$ on $J(F)$ is conjugate to the shift map on the quotient space $\Sigma^{3}$.

Proof. Recall that $A$ is the annulus bounded by $\beta$ and $\tau$, i.e. the closed annulus between the basin of $\infty$ and the trap door. Let $s=\left(s_{0} s_{1} s_{2} \ldots\right) \in \Sigma^{3}$ and recall that

$$
I_{s_{0} s_{1} \ldots s_{n}}=\left\{z \in A \mid z \in I_{s_{0}}, F(z) \in I_{s_{1}}, \ldots, F^{n}(z) \in I_{s_{n}}\right\} .
$$

As we have seen, $I_{j}$ is a closed set that is homeomorphic to a closed disk for each $j$. We also have

$$
I_{s_{0} s_{1} \ldots s_{n}} \subset I_{s_{0} s_{1} \ldots s_{n-1}} \subset \cdots \subset I_{s_{0}}
$$

for each $n$. If $F$ were one-to-one on each $I_{j}$, then we would have a well defined branch of

$$
F^{-n}: I_{s_{n}} \rightarrow I_{s_{0} s_{1} \ldots s_{n}}
$$

and this map would then be a contraction in the Poincaré metric on these spaces. Standard arguments would then show that

$$
\bigcap_{n=0}^{\infty} I_{s_{0} s_{1} \ldots s_{n}}
$$

is a unique point with the given itinerary, and this would then give the conjugacy.

Unfortunately, $F$ is not one-to-one on $I_{j}$. However, the only places where this fails is on the portions of the boundary of $I_{j}$ that meet the critical circle and the real axis. We remedy this situation as follows.

For clarity, let us restrict to $I_{0}$; the other cases are similar. Consider the subsets of $I_{0}$ of the form $I_{0 j}$ (see figure 13). We now stipulate that $I_{0 j}$ corresponds to a closed subset of $I_{0}$ that meets the interior of $I_{0}$, i.e. this set itself has interior. This eliminates certain intervals from $I_{0 j}$ along the real axis that previously made up portions of $I_{0 j}$. In particular, every point in $\mathbb{R} \cap I_{0}$ now belongs to a unique $I_{0 j}$. So the map $F: I_{0 j} \rightarrow I_{j}$ is now one-to-one on $\mathbb{R}$.

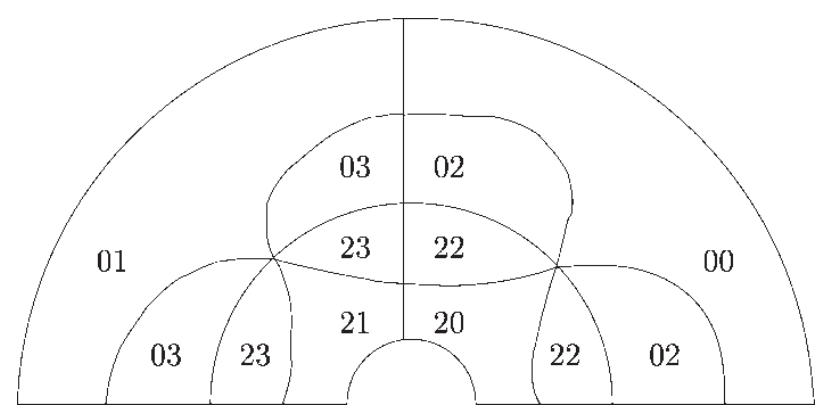

Figure 13. Some of the subsets $I_{\alpha \beta}$. 
Next note that $I_{02}$ and $I_{03}$ now consist of a pair of closed disks that are joined at a single critical point. Preimages of these sets have a similar structure. On the other hand, both $I_{00}$ and $I_{01}$ are now homeomorphic to a closed disk. So, we now adopt the convention that $I_{02}$ is one of these two closed disks. That is, two sets bear the name $I_{02}$. We make the same convention for other sets $I_{s_{0} s_{1} \ldots s_{n}}$ that are similarly joined at isolated points. This presents no ambiguity since $F$ maps one of the sets $I_{02}$ onto the portion of $I_{2}$ lying in the right half plane and $F$ maps the other set $I_{02}$ to the left half plane. Hence, one of the sets $I_{02}$ contains only $I_{022}$ and $I_{020}$ while the other contains only $I_{021}$ and $I_{023}$. That is, the subsequent digit in the sequence $s$ determines which disk to consider in the chain $I_{s_{0} s_{1} \ldots s_{n}}$. The set $I_{03}$ has similar properties.

With this specification of $I_{s_{0} s_{1} \ldots s_{n}}$, it now follows that $F^{n}: I_{s_{0} s_{1} \ldots s_{n}} \rightarrow I_{s_{0}}$ is one-to-one for each $n$. This completes the proof.

\section{Acknowledgements}

We thank the referees for numerous suggestions and simplifications concerning this paper.

\section{References}

[1] Blanchard, P., Devaney, R.L., Look, D.M., Seal, P. and Shapiro, Y. Sierpinski curve Julia sets and singular perturbations of complex polynomials. To appear in Ergodic Theory and Dynamical Systems.

[2] Blanchard, P., Devaney, R.L., Look, D.M., Moreno Rocha, M., Seal, P., Siegmund, S. and Uminsky, D. Sierpinski carpets and gaskets as Julia sets of rational maps. To appear.

[3] Devaney, R.L., 2004, Cantor and Sierpinski, Julia and Fatou: complex topology meets complex dynamics. Notices of the American Mathematical Society, 51(1), 9-15.

[4] Devaney, R.L., Look, D.M., Uminsky, D. The escape trichotomy for singularly perturbed rational maps. To appear.

[5] Douady, A. and Hubbard, J., 1982, Itération des polynômes quadratiques complexes. Comptes Rendus de l' Academie des Sciences, Paris, t.29(Serie I), 123-126.

[6] McMullen, C., 1988, Automorphisms of rational maps. Holomorphic Functions and Moduli, Vol. 1. Math. Sci. Res. Inst. Publ. 10 (New York: Springer).

[7] Milnor, J., 1999, Dynamics in One Complex Variable (Braunschweig: Vieweg).

[8] Milnor, J. and Tan, Lei, 1993, A "Sierpinski Carpet" as Julia set. Appendix F in geometry and dynamics of quadratic rational maps. Experimental Mathematics, 2, 37-83.

[9] Whyburn, G.T., 1958, Topological characterization of the Sierpinski curve. Fundamenta Mathematice, 45, $320-324$.

[10] Wittner, B., 1988, On the bifurcation loci of rational maps of degree two, Thesis, Cornell University. 